\title{
Presence for Design: Conveying Atmosphere through Video Collages
}

\author{
IANUS KELLER and PIETER JAN STAPPERS
}

\begin{abstract}
Product designers use imagery for inspiration in their creative design process. ${ }^{1}$ To support creativity, designers apply many tools and techniques, which often rely on their ability to be inspired by found and previously made visual material and to experience the atmosphere of the user environment. Computer tools and developments in VR offer perspectives to support this kind of imagery and presence in the design process. But currently these possibilities come at too high a technological overhead and price to be usable in the design practice. This article proposes an expressive and technically lightweight approach using the possibilities of VR and computer tools, by creating a sketchy environment using video collages. Instead of relying on highly realistic or even "hyperreal" graphics, these video collages use lessons learned from theater and cinema to get a sense of atmosphere across. Product designers can use these video collages to reexperience their observations in the environment in which a product is to be used, and to communicate this atmosphere to their colleagues and clients. For user-centered design, video collages can also provide an environmental context for concept testing with prospective user groups.
\end{abstract}

\section{INTRODUCTION}

$\mathbf{T}$ CAPTURE THEIR IMAgINATION, designers currently use a variety of methods, such as sketches, collages and mock-up models. ${ }^{2}$ These methods allow the designer to create, organize, and communicate rich visual media. As the interaction and complexity of products increases, designers also need more narrative tools to capture, organize, and communicate the way products are used. For this, designers have started to use methods used in theater and cinema such as role-playing, scenarios, and storyboards in their design process. ${ }^{3}$

We believe that new tools and developments in VR offer possibilities to support and even ex- tend the methods that designers use right now, if they offer good interaction, usability, and aesthetics. ${ }^{4}$ But current VR systems, though very impressive technologically and perceptually, do not sufficiently support the fluency and interactivity needed to inspire the designers in their creative process. Especially the technical overhead of creating applications, setting up the system, and calibrating the system for the user $^{5}$ keep these VR systems from being a suitable alternative to the traditional tools for inspiration. ${ }^{6}$ This situation is definitely improving, but still far from acceptable for conceptual design tools.

In our research we explore the possibilities of new inspirational tools by offering a possi-

Industrial Design Engineering, Delft University of Technology, Delft, The Netherlands. 
ble solution and using it in a given design process. This paper reports experience in constructing video collages to elicit the feeling of presence in a product's use context. The discussion here focuses on the concepts and early observations. We look at the role of the video collage as an inspirational tool in the design process, but also at how the act of making these video collages can help the designer explore the design issues related to the user environment. Formal evaluations of the proposed solutions are being conducted, and will be presented in future publications.

\section{INSPIRATION AND CONTEXT}

Imagine you are a designer being asked to design a new interactive device for consumers to be used in the kitchen. How would you take this description as a starting point for a meaningful product design? Aside from the technical issues, the designer needs to address the interaction, usability, and aesthetics of the concept. A creative solution for this requires the designer to find inspiration for this assignment. This is the main focus of our research on tools and methods in the conceptual phase of design.

The term User-Centered Design (UCD) implies an emphasis on the human user-his or her perceptual, motor, and cognitive skills. A user-centered approach usually relies heavily on user participation and extensive user testing, to develop a product that best fits the user's need. ${ }^{7}$ Presence is a person's ability to experience a certain location or context. From the per- spective of designers seeking presence, aspects of "atmosphere" are more important than any realistic or geographic location. This is similar to the use of the term "presence" in cinema or theatre. $^{8}$

The conceptual phase of design (or ideation phase) is the initial phase where designers discuss and explore their ideas, using many different methods to visualize them. ${ }^{9}$ Observations of the designer's workspace show that some of these visualizations are pasted on the walls and periphery of the workspace to serve as a source of inspiration in the creative process. ${ }^{2}$

The traditional tools designers use for inspiration are shown in Fig. 1; sketches, collages, and rough mockup models. These tools and techniques are the most widely used by designers as they support imagery, associative thinking and imagination skills, without high technical overhead and with readily available materials such as pens, glue, clay, foam, and paper.

Sketches are useful to create, explore, and communicate shape and appearance of a design and are the most widely used tools for idea creation. ${ }^{10}$ Collages are an intellectual and aesthetic exercise made out of found material to express an idea or feeling. ${ }^{11}$ The collage offers the ability to deal with design precedents and precedent knowledge and to transfer abstract ideas and concepts into a visual language or atmosphere, hardly expressible in words. ${ }^{12}$ Rough mockups, quickly made out of foam, clay, or cardboard are often used in the conceptual phase to physically experience the scale, feeling, and handling of a design.
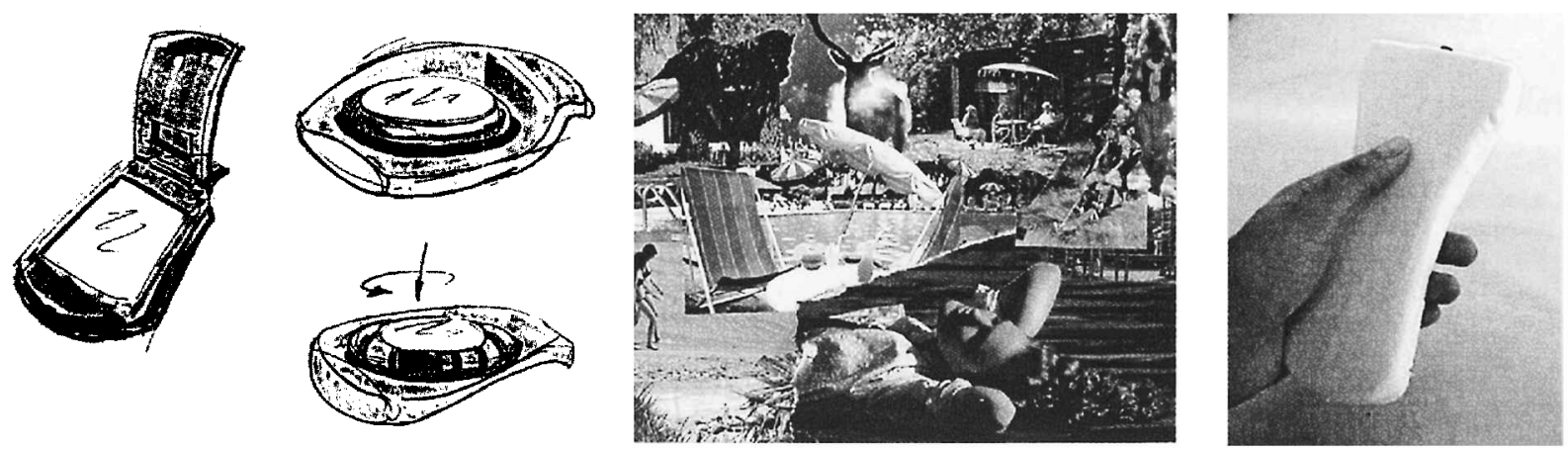

FIG. 1. Examples of the traditional tools: a sketch exploring different details, a collage showing the atmosphere of leisure time, and exploring handling in a mockup. 
In the past decade designers have started to borrow tools and methods from the field of theater and cinema (such as role-playing, scenarios, and storyboards exemplified in Fig. 2) to explore and communicate dynamic and interactive aspects of their concepts. For example, role-playing and scenarios are ways for the designers to freely explore the whole context of a product and the role it can have in the life of a user. $^{13,14}$ Designers use storyboards to look more in detail at the dynamic use of a design and the possible user interactions.

The tools and methods for inspiration mentioned above do not rely on realistic fidelity ${ }^{6,8}$; fluency, exploration and aesthetics are the more important aspects.

New tools and techniques for computer interaction and visualization can provide a more efficient and flexible way to create a sense of presence in the user environment. The technological advances allow us to create images with such a high level of detail and quality of shading, rendering, and reflections that the quality is often referred to as "hyperreal." However, these advanced VR systems lack the flexibility and ease of use that designers appreciate in their pictures, sketches, and collages. ${ }^{4}$ For example, a new concept sketch can be made in minutes; a VR simulation can take months of programming.

In general the advantages of traditional tools such as sketches, paper collages, and models can be found in the aspects of interaction, usability, and aesthetics (i.e., they allow for fluent, direct, and shared interaction, their usability allows for improvisation, and the materials used are readily available and allow for an expressive and suggestive aesthetic focusing the attention of the viewer).

Current VR tools fall short in these aspects, but when they are improved on, they can also offer advantages in simulated use and handling, easy change of environments, and the application of rich media such as sound and animation.

Although the possibilities of such new tools are promising and have shown useful achievements, the latter usually come at a price that conflicts with the demands of our simple traditional tools. The key advantage that VR-related tools offer us in the conceptual phase of design is the ability to create an immersive sense of presence. We will show that this can also be achieved with less technical overhead.

\section{THREE RANGES OF INTERACTION SETUP}

With the experimental setup TRI, or "Three Ranges of Interaction," we have tried to create a medium and an environment that allows the new possibilities offered in VR, while keeping as many of the advantages of traditional tools as possible. The TRI setup is a body-scaled interactive medium supporting the designer exploring design concepts. The term "Three Ranges of Interaction" refers to three scales in which we interact with our environment: large, medium, and small for, respectively, atmosphere, layout, and precision.
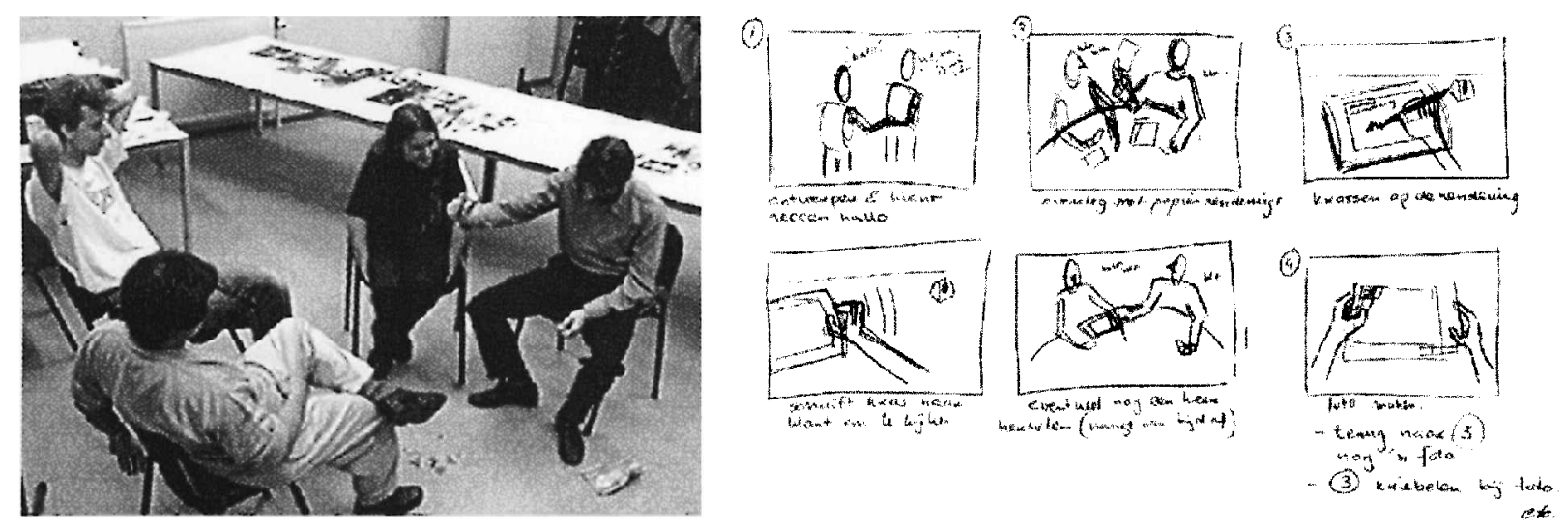

FIG. 2. Examples of tools from theater: designers doing a role-play and a storyboard for a sketching device. 


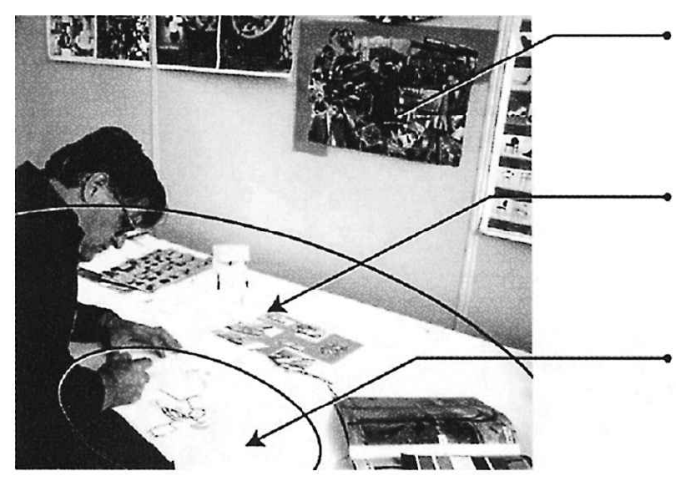

Atmosphere (large range)

Hanging collages, sketches, posters and

other sources of inspiration on the wall.

Layout (medium range)

Organizing and comparing ideas and previous concepts on the desk.

Precision (small range)

Creating and exploring concepts with sketches and models.

FIG. 3. Three ranges in the designer's work environment.

These ranges can best be illustrated by the way product designers interact with their work environment, as exemplified in Fig. 3. For atmosphere designers hang pictures, collages, and sketches of the environment of use on the walls around them. For layout, sketches and models are laid out on desks to compare and organize possible solutions and use them in discussions and presentations. Finally, for precision the designer uses paper, pens, and models to create detailed sketches.

TRI supports all three ranges with an interactive setup that provides atmosphere (large range), virtual and physical organization and presentation (medium range), and computersupported sketching (small range). The combination of the three ranges allows for an atmosphere fit to the assignment where designers can collaboratively discuss, arrange, and visualize ideas.
These three psychologically meaningful ranges are physically implemented in the TRI setup, shown in Fig. 4. By physically dividing the three ranges in the setup, content can be developed separately for the three ranges. This simplifies the development of content as the developer only has to tune and tweak one kind of interaction at the time, without influencing the other ranges at the same time. Argumentation of the design of TRI has been published online at DCNet. ${ }^{15}$ For the present argument, it is important to note that TRI attempts to make a "sketchy" form of VR, sacrificing technical fidelity for conceptual usability. Like a pencil-sketch, TRI applications aim to give a quick, rough idea rather than a fully tuned, pretty picture.

In this paper we look at how designers can use the TRI setup for inspiration by generating presence in an immersive environment. With

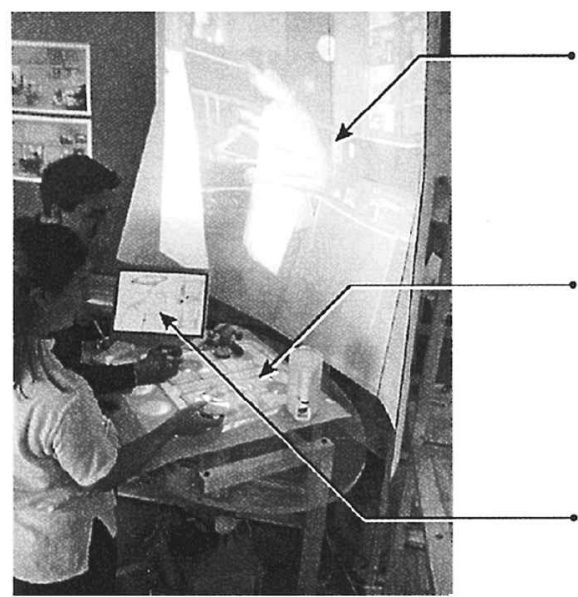

Vertical projection area (large range)
Curved cardboard screen for surround
projection of video collages.

Horizontal table projection (medium range) Table with projection for arrangement of physical and virtual projected objects.

Wacom tablet with screen (small range)

Pen input for conceptual sketching and drawing.

FIG. 4. Three scales applied in the TRI setup. 
the traditional tools this is achieved by surrounding the walls of the desk with sketches, collages, and storyboards. In contrast the TRI setup can use its large scale to serve as a rich and immersive environment, to experience the location or context of the design and its use. To create these rich environments we are exploring the use of video collages presented on the large range of the setup: a curved vertical projection area.

\section{VIDEO COLLAGES}

Video collages are expressive combinations of images sequences, video, animations, music, and sounds to communicate an atmosphere, context, or visual language. The goal of using these collages in the design process is to evoke a sense of presence in an inspiring environment related to the product to be designed. The video collages made on the TRI setup have the same sketchy aesthetics as those in paper-and-pencil sketches, cardboard mock-ups, and collages. This allows the designer to "fill in the gaps" and create his or her own interpretation of the atmosphere, which promotes active participation.

The goal of these collages is therefore not to aim for "suspension of disbelief" in a passive sense, but to use the "visceral eye" as described by Boorstin ${ }^{16}$ to help the designer who is actively wanting to participate in the simulated environment. In his book Boorstin explains that unframed images like IMAX and 3D images like the CAVE stand in the way of this visceral eye because they do not force the viewer to the center of interest. Instead of relying on picturepretty images, Boorstin advises us to use narrative, pace, montage, sound, and movement to create a compelling atmosphere. This is similar to how designers prefer to use sketches over photographs to focus the viewer's attention on the important aspects of a product design. The video collages are made using simple 2D authoring tools such as Macromedia Director or video editing suites. This low-tech approach makes it easy for the designer to focus on the content and quality of the experience, without having to communicate these ideas to a programmer or developer.

As an example of a video collage and its use, think back to the example of the designer being asked to create a product to be used in a kitchen. A good way to start the design process is to observe a kitchen environment and to capture it in a video collage. For this example we created a video collage that conveys the atmosphere of cooking a meal in a kitchen environment. The video collage uses photographs of a woman in a kitchen environment (see Fig. 5).

As shown in Fig. 5, the collage does not show a true representation of the actions in a kitchen but has the aesthetics resembling those in conventional sketches and collages. The composition of the kitchen background is created from different pictures placed together to match the visual field on the TRI setup. This creates a somewhat distorted view of reality similar to the photographic collages of David Hockney. The composition allows the designer to fill in the gaps of reality and enrich the images with his or her own experiences. ${ }^{17}$
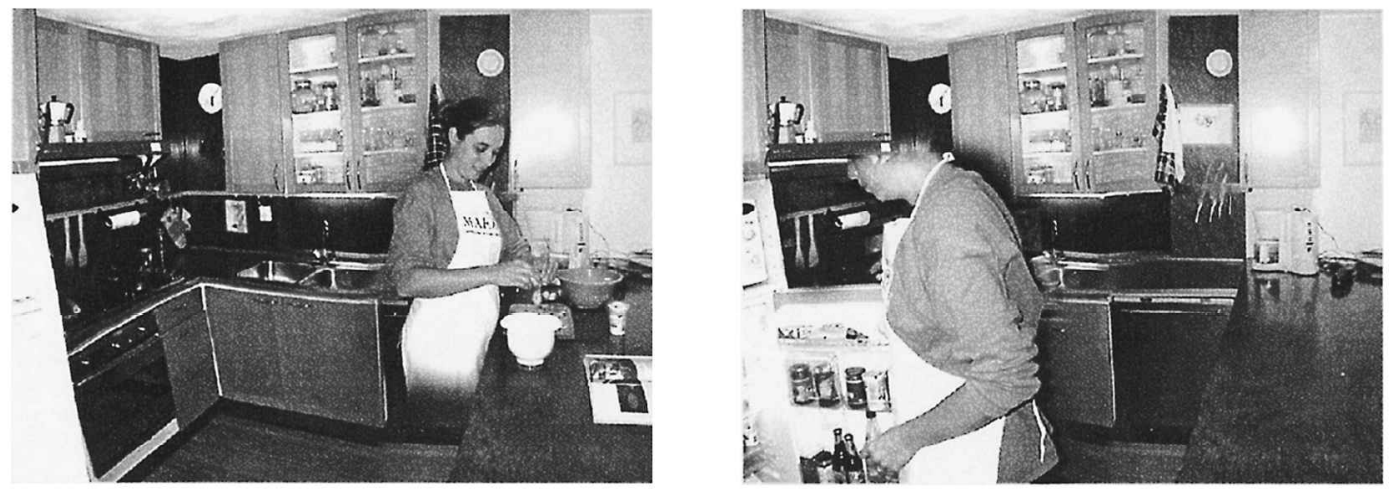

FIG. 5. Stills from the kitchen environment video collage, as presented on the vertical projection area (see Fig. 4). 
The different actions in the kitchen (such as cutting, baking, washing) are displayed as sequences of still photo inserts and recorded sound. The selection of the photo inserts highlights the actions, cutting out many irrelevant parts of reality such as the legs or head of the woman in the kitchen. In this way the kitchen stays completely clean and unchanged at all times with only a relevant change in the area of the woman's actions. This method of animation provides a clear focus of attention on the actions of the woman in the kitchen.

The use of stop motion allows the author of the video collage to balance the timing of the different actions in the kitchen. Sounds that accompany the actions serve as a continuous and ubiquitous reminder of the context even when the attention is not focused on the projection.

The sketchy aesthetics of the video collage encourages the same participation by the designers as conventional sketches and collages do. The use of stop motion exaggerates the actions of the user, so that it forces the viewer to the center of interest. ${ }^{18}$ These forms of incompleteness, as compared to plain video, promote the designer's participation in the environment (i.e., his or her sense of presence).

\section{VIEWING VIDEO COLLAGES FOR INSPIRATION}

We conducted a study to compare the effect of traditional collages and video collages in the design process. The goal of this study was to find out which part of the different types of collages the designers pick up in their design activity. The hypothesis was that designers pick up more form- and color-related aspects from the traditional collages and more contextual, interactive and usage aspects from the video collages.

Two groups of design students were asked to sketch a concept for an MP3 player. One group used a video collage showing the use environment, possible users, and form details as inspiration. The other group relied on a traditional collage providing similar information. After they completed their concept sketches they were interviewed as to the use of the collage, the experience of the environment, and the elements of the collage that influenced their designs.

Though we used a small group (five students) to test our hypothesis on, we found that all our subjects readily accepted the video collage as a useful way to communicate context and atmosphere, and focused more on the large concept of usability and context of the design.

We present here only the observation rather than a formal description of the experiment, which was limited in scope. For instance, in this study, the collages were prepared by the experimenting researchers, not by the participating design students. In the conceptual phase of design the act of making collages, sketches, models and so forth is at least as important as the end result itself. Therefore we are actively studying the creation process itself as well, and a new experiment is planned to further validate these findings.

\section{MAKING VIDEO COLLAGES FOR INSPIRATION}

To experience the possibilities that the TRI setup has to offer in creating an atmosphere, several video collages have been made by 12 design masters students of different user contexts. In this aesthetic exercise they focused on the cinematic aspects of the video collage that influence the atmospheric experience: narrative, framing, timing, transitions, sound, perspective, and lighting. Moreover, the students explored the usefulness of creating video collages in the conceptual phase of the design process.

All the video collages were made in a relatively short time-varying from 4 to 12 hoursand required setting out a design goal, choosing an environment, observing the aspects of atmosphere, capturing these observations in sound and images, and combining them in a video collage.

The 12 different video collages offer many different experiences: from being in a busy marketplace to a quiet beach to sitting in the chair at the dentist's. The feedback given by the students on their video showed that they found it very important to set the goals on conveying an atmosphere opposed to just creating a pretty 
image or good sound. The students who took the longest time tweaking aspects of sound quality or image quality in itself were the least satisfied with their results, whereas the students that took more time trying out the complete experience and overall narrative achieved the most useful and aesthetically expressive results, which will be published on our TRI Web site.

\section{USING VIDEO COLLAGES FOR CONCEPT TESTING}

The conceptual phase of design relies heavily on iterations, trying out different concepts and verifying them with the end users. Therefore almost all guidelines on user-centered design stress the importance of testing ideas with users in the earliest possible phase. ${ }^{19}$ The problem with most of these informal tests is that it's hard to test a concept in the right atmospheric contexts.

With video collages on the TRI setup it is possible to quickly and easily convey a sense of atmosphere as a backdrop for user testing. When the TRI setup is used for this purpose the video collage on the large range is for conveying the atmosphere and context of the use environment of the product. We have used video collages on the TRI setup in a series of interface design evaluations.

In one experiment an interface for a portable navigation aid running on a handheld com- puter was tested. The application is intended to be used while walking through a city. The interface design was simulated on a normal PC with a touchscreen, which made it impossible to test the concept in the field. The original solution was to provide a sense of context in another part of the same touchscreen (see Fig. 6). As an alternative, we proposed using a video collage of the user environment projected, with the touchscreen tilted in such a position that it would resemble the way a user holds a handheld computer.

During the usability test our subjects were asked to navigate through the city by using arrow keys on the touchscreen with the navigation interface for reference. As the users navigated through the city the experimenter changed the backdrops according to the appropriate location (this could have been programmed, but for a short study, a Wizard of Oz technique, in which a human makes these decisions, is often more efficient ${ }^{20}$ ). After completing a task the users were asked to recollect some details of the projected environment. This way the experimenters could measure the amount of attention the user needs for operating the handheld computer. ${ }^{21}$

Though the usability of video collages for usability testing itself was not the goal of this experiment, we were able to verify that our users readily accepted the projection as an environment. Even without an explanation of the background or purpose of the large screen projection, the users easily accepted the video collage
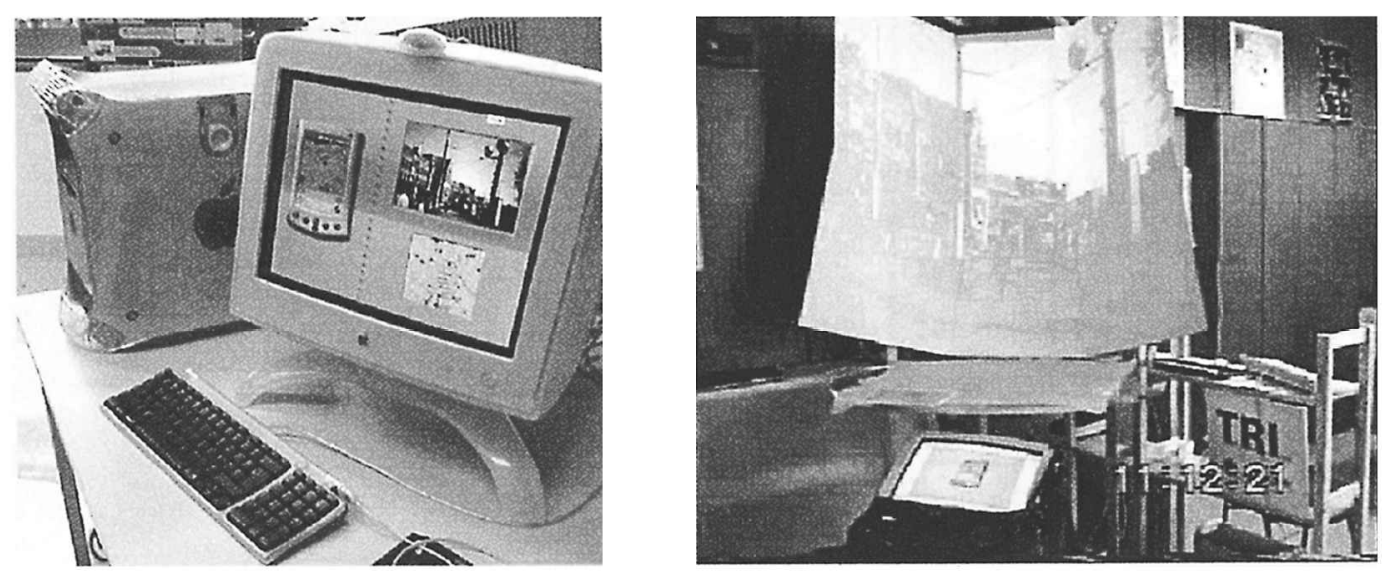

FIG. 6. Images from the original screen-based setup next to the TRI setup alternative. 
as a means to illustrate the environment of the user context.

\section{CONCLUSION}

Computer tools can provide a sense of presence that is useful for the conceptual design process. To allow the designers to suspend their disbelief, these tools will focus on easy ways to create a sense of the environment in an abstraction such as a video collage. These collages offer possibilities for abstraction and expression that designers have learned to appreciate in their pen and paper based ideation tools (sketches and collages). The TRI setup provides the tools to create sketchy representations of a user environment in video collages. Although we have not completed rigorous experiments yet, we feel confident in the following observations.

The possibilities of dynamics, interactivity, and the use of digitized sounds makes these virtual environments more engaging and flexible than the current conventional methods such as making conventional collages or filling the walls with sketches and photographs. Moreover these video collages form an important aspect of the conceptual design process as an aesthetic exercise. By using sketchy aesthetics and keeping the time to create as short as possible, the designer can explore, visualize, and communicate the user environment as an atmosphere.

Lessons learned from cinema and theater can be used to lure the attention of the viewer to important aspects of the environment. This is similar to the way the sketchiness in drawings is used to emphasize important aspects of the product design. This approach assumes that if the viewer is prepared to fill in the gaps, she can immerse herself in an environment by her own imagination and doesn't have to rely on the highly realistic, expensive and difficult to make three-dimensional graphic representations.

Designers can use these video collages for inspiration and verification. Inspiration can be promoted by creating a sense of presence in the environment in which the product will be used; verification is possible when testing a concep- tual design on users in a simulation of the user environment.

\section{ACKNOWLEDGMENTS}

The TRI setup was created in collaboration with the Academic Computing Services Amsterdam (SARA), who have shared their knowledge and experiences in the field of presence in their CAVE systems. Especially Jorrit Adriaanse of the Academic Computing Services Amsterdam has been a driving force in making the TRI setup a reality. The TRI setup is an integral part of the interior of the ID-Studiolab, a collaborative research workspace at the faculty of Industrial Design Engineering. More information on TRI and its developments can be found on our Web site at http://www.io. tudelft.nl/id-studiolab/tri/index.html. Moreover, the authors have benefited greatly from the ideas and discussions on the TRI setup with the members of the ID-StudioLab.

\section{REFERENCES}

1. Athavankar, U.A. (1997). Mental imagery as a design tool. Cybernetics and Systems, 28:25-41.

2. Kolli, R., Pasman, G.R., \& Hennessey, J.M. (1993). Some considerations for designing a user environment for creative ideation. Proceedings of INTERFACE 93. Santa Monica, CA: Human Factors \& Ergonomics Society, pp. 72-77.

3. Verplank, W., Fulton, J., Black, A., \& Moggridge, W. (1993). Observation and invention: The use of scenarios in interaction design. CHI Tutorial, New York: ACM.

4. Stappers, P.J., \& Hennessey, J.M. (1999). Towards electronic napkins and beermats: Computer support for visual ideation skills. In, Paton, R.C., Neilson, E., (eds.) Visual representations and interpretations. Proceedings of VRI'98; Berlin: Springer.

5. Krueger, M. (1995). When, why, and whether to experience virtual reality. Proceedings of Virtual Reality World'95, 477-481.

6. Stappers, P.J., Gaver, W., \& Overbeeke, K. (2001). Beyond the limits of real-time realism. Chapter to appear in: Hettinger, L., \& Haas, M., (eds.) Psychological issues in the design and use of virtual and adaptive environments. Mahwah, NJ: Lawrence Erlbaum Associates, Inc., Publishers.

7. Norman, D.A. (1988). The psychology of everyday things. New York: Basic Books.

8. Naimark, M. (1997). What's wrong with this picture? Presence and abstraction in the age of cyberspace. Consciousness reframed: Art and consciousness in the post- 
biological era proceedings. Ascott, R., (ed.) Newport: University of Wales College.

9. McKim, R.H. (1980). Experiences in visual thinking. Belmont, CA: Wadsworth.

10. Verstijnen, I.M. (1997). Sketches of creative discovery: A psychological inquiry into the role of imagery and sketching in creative discovery. Doctoral thesis, Delft University of Technology.

11. Lynch, J. (1961). How to make collages. Northampton: Viking Press.

12. Tufte, E.R. (1997). Visual explanations, images and quantities, evidence and narrative. Cheshire: Graphics Press.

13. Burns, C., Dishman, E., Verplank, W., \& Lassiter, B. (1994). Actors, hairdos and videotape-Informance Design. CHI'94 Conference Companion. New York: ACM Press.

14. Djajadiningrat, J.P., Gaver, W.W., \& Frens, J.W. (2000). Interaction relabelling and extreme characters: Methods for exploring aesthetic interactions. Proceedings of DIS'00, Designing Interactive Systems. New York: ACM.

15. Keller, A.I., Stappers, P.J., \& Hoeben, A. (2000). TRI: Inspiration support for a design studio environment. Proceedings of DCNet 2000, University of Sydney. Online document: http://www.arch.usyd.EDU.AU/kcdc/ journal/vol3/dcnet/keller/

16. Boorstin, J. (1995). Making movies work. Thinking like a filmmaker. Los Angeles: Silman-James.
17. McCloud, S. (1993). Understanding comics, the invisible art. Northampton: Kitchen Sink Press.

18. Wurman, R.S. (1989). Information anxiety. New York: Doubleday.

19. Nielsen, J. (1994). Usability engineering. Los Angeles: Morgan Kaufmann.

20. Gould, J.D. (1995). How to design a usable system. In: Baecker, R.M., Grudin, J., Buxton, W.A.S., Greenberg, S., (eds.) Readings in human-computer interaction: Towards the year 2000. San Francisco: Morgan Kaufmann Publishers, Inc., pp. 528-539.

21. Wolf, E., Keyson, D.V. (2000). Lokaal Mobiel Navigatiesysteem (Dutch). Unpublished internal research document, Delft University of Technology.

Address reprint requests to: I. Keller

Industrial Design Engineering Delft University of Technology Jaffalaan 9

2628 BX Delft, The Netherlands

E-mail: a.i.keller@io.tudelft.nl 